\title{
Application of multiline two-photon microscopy to functional in vivo imaging
}

\author{
Rafael Kurtz ${ }^{\mathrm{a}, *}$, Matthias Fricke ${ }^{\mathrm{b}, 1}$, Julia Kalb ${ }^{\mathrm{a}, 2}$, \\ Philip Tinnefeld ${ }^{\mathrm{b}, 3}$, Markus Sauer ${ }^{\mathrm{b}, 4}$ \\ ${ }^{a}$ Lehrstuhl für Neurobiologie, Universität Bielefeld, Postfach 100131, D-33501 Bielefeld, Germany \\ ${ }^{\mathrm{b}}$ Lehrstuhl für Angewandte Laserphysik und Laserspektroskopie, Universität Bielefeld, \\ Postfach 100131, D-33501 Bielefeld, Germany \\ Received 3 November 2005; received in revised form 28 November 2005; accepted 4 December 2005
}

\begin{abstract}
High spatial resolution and low risks of photodamage make two-photon laser-scanning microscopy (TPLSM) the method of choice for biological imaging. However, the study of functional dynamics such as neuronal calcium regulation often also requires a high temporal resolution. Hitherto, acquisition speed is usually increased by line scanning, which restricts spatial resolution to structures along a single axis. To overcome this gap between high spatial and high temporal resolution we performed TPLSM with a beam multiplexer to generate multiple laser foci inside the sample. By detecting the fluorescence emitted from these laser foci with an electron-multiplying camera, it was possible to perform multiple simultaneous linescans. In addition to multiline scanning, the array of up to 64 laser beams could also be used in $x-y$ scan mode to collect entire images at high frame rates. To evaluate the applicability of multiline TPLSM to functional in vivo imaging, calcium signals were monitored in visual motionsensitive neurons in the brain of flies. The capacity of our method to simultaneously acquire signals at different cellular locations is exemplified by measurements at branched neurites and 'spine'-like structures. Calcium dynamics depended on branch size, but 'spines' did not systematically differ from their 'parent neurites'. The spatial resolution of our setup was critically evaluated by comparing it to confocal microscopy and the negative effect of scattering of emission light during image detection was assessed directly by running the setup in both imaging and point-scanning mode.
\end{abstract}

Keywords: Two-photon; Microscopy; Calcium imaging; Temporal resolution; Beamsplitter; Visual system; Invertebrate

\section{Introduction}

The investigation of dynamic processes in single nerve cells and neuronal circuits often requires imaging neural activity and its correlatives with high temporal and spatial resolution. In conventional wide-field epifluorescence microscopy of intact tissue spatial resolution is affected considerably by light scattering. Temporal resolution, on the other hand, is limited by the

\footnotetext{
* Corresponding author. Tel.: +49 521106 5577; fax: +49 5211066038. E-mail addresses: rafael.kurtz@uni-bielefeld.de (R. Kurtz), mfricke@physik.uni-bielefeld.de (M. Fricke),julia.kalb@uni-bielefeld.de (J. Kalb), tinnefeld@physik.uni-bielefeld.de (P. Tinnefeld), sauer@physik.uni-bielefeld.de (M. Sauer).

1 Tel.: +49521 106 5434; fax: +495211062958.

2 Tel.: +49 521106 2548; fax: +49521 1066038 .

3 Tel.: +49 5211065442 ; fax: +49521 1062958 .

${ }^{4}$ Tel.: +49 521106 5450; fax: +49 5211062958 .
}

time needed to acquire image sequences with a charged-coupled device (CCD) camera under low-light conditions.

Laser-scanning fluorescence techniques, in particular TPLSM, may provide better spatial resolution in scattering tissue than conventional imaging and allow three-dimensional reconstructions based on image series at different focal planes (for review see: Halbhuber and König, 2003; Helmchen and Denk, 2002; Wright and Wright, 2002; Zipfel et al., 2003). The superior spatial resolution of laser-scanning microscopy is due to the fact, that either signal detection (in confocal microscopy) or excitation itself (in TPLSM) is confined to the laser focus within the sample.

Imaging speed, however, is a problem in laser microscopy: acquisition rate can usually be raised to several hundreds of samples per second only in line scans, i.e. when the laser is scanned repeatedly along a single axis. In contrast, scanning entire images or even image stacks in the $z$-plane is as timeconsuming as conventional wide-field fluorescence imaging. 
This bears considerable problems when comparing functional signals at several neuronal structures, which cannot be covered entirely by a single scan line. Unfortunately, non-simultaneous, consecutive data collection for several line positions is often not an adequate option, because it renders conclusions too indirect in the face of large trial-to-trial variability in neuronal responses (see e.g. Passaglia and Troy, 2004; Warzecha and Egelhaaf, 1999). Techniques which increase temporal resolution during acquisition of entire images, such as spinning disc confocal microscopy, often yield too weak signals for the application in the compact tissue of living samples, although new developments are promising (see e.g. Coates et al., 2004; Nakano, 2002).

In this study we investigate whether multiline TPLSM can help to overcome the trade-off between high temporal and high spatial resolution in functional imaging.

The principle of multiline TPLSM builds on a mirror-based beam multiplexer, which divides a single laser beam into multiple beams (Nielsen et al., 2001). In TPLSM, where excitation is intrinsically confined to the tiny focal volume of a laser, this beam multiplexer can be used to generate several of such excitation spots in the focal plane of the sample. We demonstrate how this multifocal excitation scheme can be efficiently used to acquire multiple linescans simultaneously. Furthermore, acquisition of entire images is accelerated when scanning with multiple laser foci because each beam scans only a part of the image or each sample point is excited by several beams in succession. Recently, a similar beam multiplexer design has been proposed for the use in fluorescence lifetime imaging (LevequeFort et al., 2004).

Imaging in intact animals belongs to the most challenging tasks in functional microscopy (for review see: Helmchen and Waters, 2002). We use monitoring of neuronal calcium dynamics in the visual system of the fly as a critical test for multiline TPLSM. The visual motion-processing area in the brain of the blowfly (Calliphora vicina), the lobula plate, contains about 60 large, individually identifiable, direction-selective neurons, socalled tangential cells (TCs) (for reviews see Borst and Haag, 2002; Egelhaaf and Borst, 1993; Egelhaaf et al., 1988, 2002, 2005). These neurons have become a unique model system for the study of neuronal information processing under close-tonatural conditions. In electrophysiological and optical recording experiments, TCs were shown to function as motion sensors, each one tailored in a specific way to extract distinct components of optic flow information from the dynamic patterns generated on the eyes during locomotion (Dürr et al., 2001; Egelhaaf et al., 1993; Krapp and Hengstenberg, 1996; Single and Borst, 1998). TCs have large receptive fields, due to retinotopic dendritic sampling of numerous signals from peripheral local motion-sensitive elements (Borst and Egelhaaf, 1992). In addition, interactions between TCs further increase receptive field size and motion specificity (Haag and Borst, 2001; Horstmann et al., 2000).

Calcium imaging has been used as a marker of local dendritic activation in TCs (Borst and Egelhaaf, 1992; Dürr and Egelhaaf, 1999; Haag et al., 2004; Single and Borst, 1998). Furthermore, there is evidence for an involvement of dendritic calcium accumulation in the activity-dependent regula- tion of motion sensitivity, i.e. motion adaptation (Kurtz et al., 2000). Imaging of presynaptic calcium concentration changes has been used to outline the properties of graded synaptic transfer during sensory stimulation (Kurtz et al., 2001). In all these issues, advanced spatial and temporal resolution of calcium imaging will help us to draw more direct conclusions, e.g. on the properties of dendritic input integration, on the dynamic calcium regulation during synaptic transmission, and on the potential spatiotemporal control of motion adaptation by calcium.

TPLSM is the method-of-choice for in vivo imaging, in particular in the visual system, since in addition to its superior performance and reduced photodamage during excitation in deeper layers of compact tissue, visual excitation of the photoreceptors is avoided due to the use of near-infrared wavelengths (see e.g. Denk and Detwiler, 1999; Euler et al., 2002). In contrast, both confocal and conventional imaging is often accompanied by unwanted visual stimulation, because one-photon excitation spectra of fluorescent dyes largely overlap with those of photoreceptors. The fly visual system was first shown to be amenable to in vivo TPLSM by monitoring calcium signals by singleline scanning and detection with an avalanche photodiode (Kalb et al., 2004). Afterwards, Haag et al. (2004) analyzed local dendritic calcium oscillations in TCs during motion of stripe patterns, in order to draw conclusions on the computational scheme of local motion detector input of TCs. In the latter study $64 \times 64$ pixel images were acquired, but the temporal resolution of calcium imaging was only $8 \mathrm{~Hz}$. In contrast, Kalb et al. (2004) obtained a high temporal resolution of $400 \mathrm{~Hz}$ by singleline scanning, i.e. with spatial resolution restricted to one line of $10 \mu \mathrm{m}$ length. The present study demonstrates for the first time how a multiline principle in TPLSM can be efficiently applied in order to bridge the gap between temporal and spatial resolution in functional imaging of neural activity.

\section{Methods and results}

In order to draw relevant conclusions from functional imaging by laser-scanning microscopy a high temporal resolution is often essential. At low-light conditions, this can normally only be achieved by performing single-line scans. This is exemplified by calcium imaging performed with a confocal microscope (Leica SP2) at presynaptic arborizations of a 'Vertical System' (VS) neuron (see Fig. 1, Table 1). This type of TC responds to downwards motion in its receptive field with a graded depolarization of its membrane potential, which is accompanied by calcium accumulation in the fine endings of the presynaptic region (Kurtz et al., 2001). In order to reach a high temporal resolution $(400 \mathrm{~Hz})$, a single-line scan was performed. In the recording shown, it was possible to hit two neurites of different diameters with the scan line. Increases in the fluorescence of the calcium indicator dye Oregon-Green 488 BAPTA-1 relative to baseline fluorescence $\left(\Delta F / F_{0}\right)$ during pattern motion indicated a rise in the concentration of free intracellular calcium. The calcium signal at the thin neurite appeared faster in both its rise during stimulus motion and its decline following the cessation of stimulus motion. 


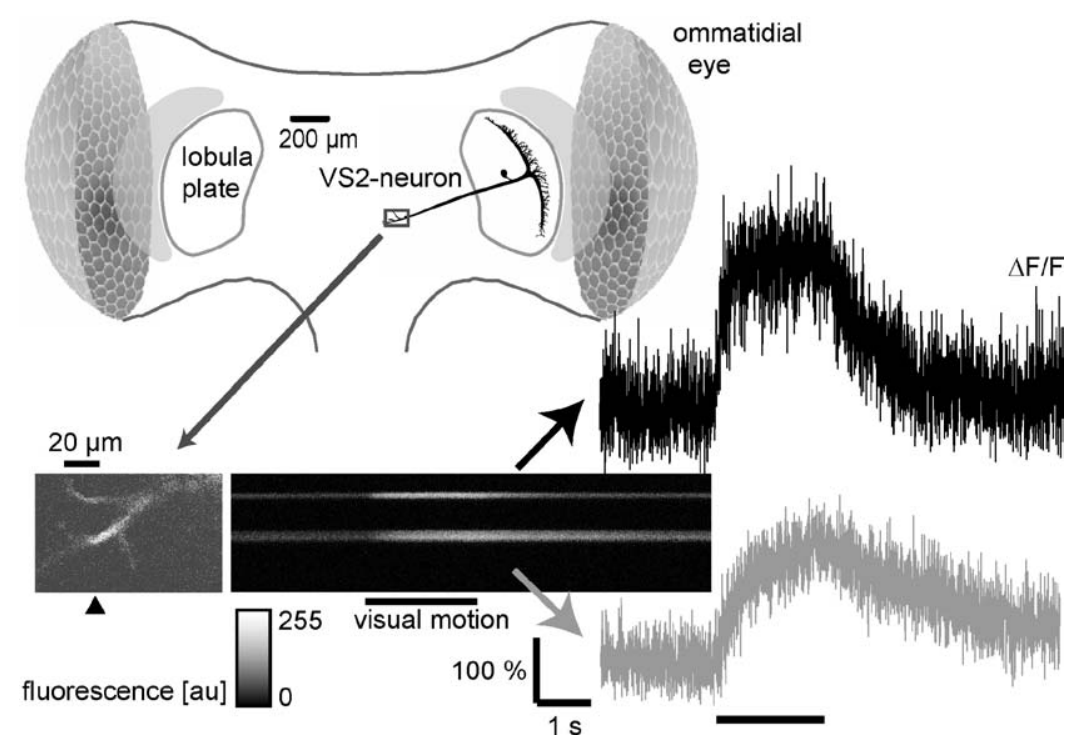

Fig. 1. Top: schematic of the fly's head (caudal view). Vertical System (VS) neurons form an ensemble of 10 cells, belonging to the visual motion-sensitive tangential cells in the fly brain. To study calcium concentration changes in these neurons the lobula plate region was exposed, a single VS2-neuron was impaled with a sharp glass electrode for intracellular recordings and the electrically charged, membrane-impermeant calcium dye Oregon-Green 488 BAPTA-1 was driven into the cell by applying hyperpolarizing current ( -1 to $-2 \mathrm{nA}$ for 3-5 min). Bottom: single-line scans were performed at the presynaptic region of the VS2-neuron with a Leica SP2 confocal microscope equipped with a long-distance water-immersion $40 \times /$ N.A. 0.8 objective (excitation $488 \mathrm{~nm}$, emission $500-560 \mathrm{~nm}$, scan frequency $400 \mathrm{~Hz}$ ). The arrow below the image of the presynaptic arborizations (left) indicates line position. During downwards motion of a squarewave grating (spatial wavelength: $32^{\circ}$, temporal frequency: $4 \mathrm{~Hz}$, Michelsen contrast: $99.3 \%$ ) intracellular free calcium rised, leading to an increase in dye fluorescence (middle). The time course of relative fluorescence changes $\left(\Delta F / F_{0}\right)$ is shown averaged over regions containing signal from the upper, thinner neurite (right, black trace) and the lower, thicker neurite, respectively (grey trace). Both the rise of the calcium signal during pattern motion and its decline afterwards appeared faster in the thin neurite. For a more detailed description of animal preparation, electrophysiological procedures and visual stimulation see Kurtz et al. (2001).

A systematic analysis of the differences in calcium dynamics between individual neurites by single-line scanning in highly branched structures, such as dendrites and presynaptic arborizations is difficult, because it is hardly controllable, whether more than one area of interest is hit by the line. To solve these problems to some extent, we present a method to perform calcium imaging by TPLSM with multiple laser beams instead of a single beam.

\subsection{Principle of multiline TPLSM}

Pulsed near-infrared laser light from a tunable Ti:sapphire laser (see Table 1) was directed to a mirror-based beam multiplexer (Nielsen et al., 2001). The principle of the beam multiplexer is to combine a central 50\%-beamsplitter mirror with high-reflectivity mirrors to both of its sides (see Fig. 2). The beamsplitter mirror provided doubling of incoming beams and the high-reflectivity mirrors sent the beams repeatedly back to the central beamsplitter mirror. This procedure generated an array of up to 64 laser beams arranged in a row.

The beam multiplexer used in our initial experiments (see also Kalb et al., 2004) consisted of an arrangement of single optical components, each mounted and adjusted directly on a vibrationfree table. In later experiments (on which all data shown here are based), this custom-built setup of the beam multiplexer was, for reasons of convenience, replaced by a recently developed commercially available version (TriM-Scope, LaVision BioTec, Bielefeld, Germany). The latter comprises essential components of the setup, namely attenuator, beam telescope, disper- sion compensation, beam multiplexer, and $x y$-galvo-scanners, all mounted inside a single housing.

After passing the scanners, the array of laser beams entered an inverted microscope (Olympus IX70) via its backport. A fly with its brain exposed and a single TC filled with calcium-sensitive dye for fluorescence imaging was mounted on the microscope stage. An LED board for presentation of visual stimuli was placed in the fly's visual field.

\subsection{Emission-light detection by imaging in multiline TPLSM}

In single-beam TPLSM, photomultiplier tubes (PMTs) or avalanche photodiodes (APDs) are conventionally used as emission light detectors. Due to the principle of two-photon illumination, excitation is confined to the laser focus. Thus, a confocal pinhole to block out-of-focus light is unnecessary, and nondescanned detection can be used. That means, excitation light is not send back to the scan mirrors, but directed straight to a PMT with a large active area, positioned close to the objective lens. Such a simple detection mode can, however, hardly be realized with multiline TPLSM, because here a separation of the fluorescence originating from each of the laser foci is necessary. Therefore, we first envisaged a detection scheme with multiple APDs operated in descanned mode. The sensitivity of this detection mode was tested with positive results in preliminary experiments with only one laser beam and a single APD (Kalb et al., 2004). However, we refrained from installing additional APDs for multiline detection, because detector adjustment 
Table 1

Summary of materials and equipment

\begin{tabular}{|c|c|c|}
\hline Equipment item & Specifications & Manufacturer \\
\hline \multicolumn{3}{|l|}{ I. Electrophysiological procedures } \\
\hline Insect ringer's solution & $\begin{array}{l}\text { (in mM) } 1.9 \mathrm{CaCl}_{2}, 13.9 \text { glucose, } 5.4 \mathrm{KCl}, 3.4 \mathrm{KH}_{2} \mathrm{PO}_{4} \text {, } \\
128.3 \mathrm{NaCl}, 4.8 \mathrm{NaHCO}_{3}, 3.3 \mathrm{Na}_{2} \mathrm{HPO}_{4}(\mathrm{pH} 7.0)\end{array}$ & $\begin{array}{l}\text { All from Merck (Darmstadt, Germany), except: } \mathrm{KCl} \text { : } \\
\text { Sigma (Deisenhofen, Germany) }\end{array}$ \\
\hline Electrode solutions & $\begin{array}{l}\text { Shaft filling: (in M) } 1 \mathrm{KCl} \text {; tip filling (data for Fig. 1): } \\
\text { (in mM) } 33.3 \mathrm{KCl}, 1.7 \mathrm{KOH}, 33.3 \mathrm{Hepes}(\mathrm{pH} 7.3) 17\end{array}$ & $\begin{array}{l}\text { All from Merck (Darmstadt, Germany), except: } \mathrm{KCl} \text {, } \\
\text { Hepes: Sigma (Deisenhofen, Germany) Ca-dyes: }\end{array}$ \\
\hline & $\begin{array}{l}\text { Oregon-Green } 488 \text { BAPTA-1 hexapotassium salt; tip } \\
\text { filling (data for Fig. 3): (in } \mathrm{mM} \text { ) } 33.3 \mathrm{KCl}, 1.7 \mathrm{KOH} \text {, } \\
33.3 \text { Hepes (pH 7.3), } 17 \text { fluo-4 pentapotassium salt, } 2.3 \\
\text { Oregon-Green } 488 \text { BAPTA-1 hexapotassium salt }\end{array}$ & Molecular Probes (Eugene, OR) \\
\hline Electrode glass capillaries & Borosilicate glass GC100TF-10 & Clark Electromedical (Edenbridge, UK) \\
\hline Electrode puller & Brown-Flaming P97 & Sutter Instruments (San Rafael, CA) \\
\hline Intracellular amplifier & Axoclamp $2 \mathrm{~A}$ & Axon Instruments (Foster City, CA) \\
\hline \multicolumn{3}{|l|}{ II. Visual stimulation } \\
\hline LED board & $\begin{array}{l}\text { Rectangular board with } 1440 \text { green } 2.5 \mathrm{~mm} \times 4.8 \mathrm{~mm} \\
\text { LEDs ( } 48 \text { rows, } 30 \text { columns) }\end{array}$ & $\begin{array}{l}\text { Custom-built (Electronics Workshop, Bielefeld } \\
\text { University) }\end{array}$ \\
\hline $\mathrm{AD} / \mathrm{DA}$-converter & DT2801A & Data Translation (Marlboro, MA) \\
\hline \multicolumn{3}{|l|}{ III. Confocal imaging } \\
\hline Confocal microscope & TCS SP2 & Leica Microsystems (Mannheim, Germany) \\
\hline Microscope objective & $\begin{array}{l}\text { Long-distance water-immersion HCX APO L UV-I } \\
40 \times / 0.80\end{array}$ & Leica Microsystems (Mannheim, Germany) \\
\hline Z-piezo & P-721.10 PIFOC & Physik Instrumente (Karlsruhe, Germany) \\
\hline Z-piezo controller & E-662.LR & Physik Instrumente (Karlsruhe, Germany) \\
\hline \multicolumn{3}{|l|}{ IV. Multiline TPLSM } \\
\hline $\begin{array}{l}\text { Tunable pulsed femtosecond } \\
\text { titanium:sapphire IR laser }\end{array}$ & $\begin{array}{l}\text { MaiTai, wavelength, } 780-920 \mathrm{~nm} \text {; pulse width }<100 \mathrm{fs} \text {, } \\
\text { repetition rate, } 80 \mathrm{MHz} \text {; average power, } 650-1100 \mathrm{~mW} \\
\text { (wavelength-dependent) }\end{array}$ & Spectra-Physics (Mountain View, CA) \\
\hline $\begin{array}{l}\text { Mirror-based beam } \\
\text { multiplexer }\end{array}$ & $\begin{array}{l}\text { TriMScope, including attenuator (step-motor driven } \lambda / 2 \\
\text { plate with thin-film polariser), prismatic dispersion } \\
\text { compensation, beam telescopes }\end{array}$ & LaVision BioTec (Bielefeld, Germany) \\
\hline $\begin{array}{l}\text { Nonresonant galvanometer } \\
x y \text {-scanners }\end{array}$ & VM 500 with Mini-SAX controller & GSI Lumonics (Billerica, MA) \\
\hline Vibration-free table & $\begin{array}{l}\text { RP reliance sealed hole table top on four laminar flow } \\
\text { isolators PL- } 2000 \text { series }\end{array}$ & Newport (Mountain View, CA) \\
\hline Inverted microscope & IX-70 & Olympus (Melville, NY) \\
\hline Microscope objective & $\begin{array}{l}\text { Long-distance water-immersion LUMPlanFl/IR } \\
40 \times / 0.80\end{array}$ & Olympus (Melville, NY) \\
\hline Microscope objective & Water-immersion UPlanApo $60 \times / 1.20$ & Olympus (Melville, NY) \\
\hline Filters & BP $420-480$, BA 515 & Olympus (Melville, NY) \\
\hline TP beam splitter & 680DCSPXR & AHF Analysentechnik (Tübingen, Germany) \\
\hline TP-emission filter & E700SP-2P & AHF Analysentechnik (Tübingen, Germany) \\
\hline $\begin{array}{l}\text { Back-illuminated } \\
\text { electron-multiplying CCD } \\
\text { camera (EMCCD) }\end{array}$ & $\begin{array}{l}\text { iXon DV887BI, chip size, } 512 \times 512 \text { pixels; read out } \\
\text { rate, } 10 \mathrm{MHz} \text { at } 14 \text { bit }\end{array}$ & Andor Technology (Belfast, Northern Ireland) \\
\hline \multicolumn{3}{|l|}{ V. Sensitivity and resolution tests } \\
\hline Quantum dots & Qdot 605 streptavidin conjugate & Quantum Dot Corporation (Hayward, CA) \\
\hline Fluorescent microspheres & TransFluoSpheres 488/685, $40 \mathrm{~nm}$ & Molecular Probes (Eugene, OR) \\
\hline
\end{tabular}

Note: The authors have no affiliations with any of the manufacturers listed above. Our choice of equipment items is not meant to imply, that they are superior to similar products from other suppliers.

turned out to be critical. Instead, prompted by the advent of ultrasensitive CCD cameras with on-chip signal amplification (so-called electron-multiplying CCDs, EMCCDs), we decided to use imaging detection in non-descanned mode. In this mode, entire images are acquired by sychronizing $\mathrm{CCD}$ read-out to one or multiples of sweeps of the laser beams across the samples. This is in contrast to point-scanning detection with PMTs or APDs, where one value is sampled for every position of the laser focus and the image is reconstructed by relating these values to the laser positions. In scattering tissue the use of imaging detection instead of point-scanning detection will inevitably lower spatial resolution to some extent. As we show later, multiline TPLSM can also be used with point-scanning detection, although at a much lower temporal resolution than with imaging detection (see later this section and Section 3 for a critical evaluation of both detection schemes). 


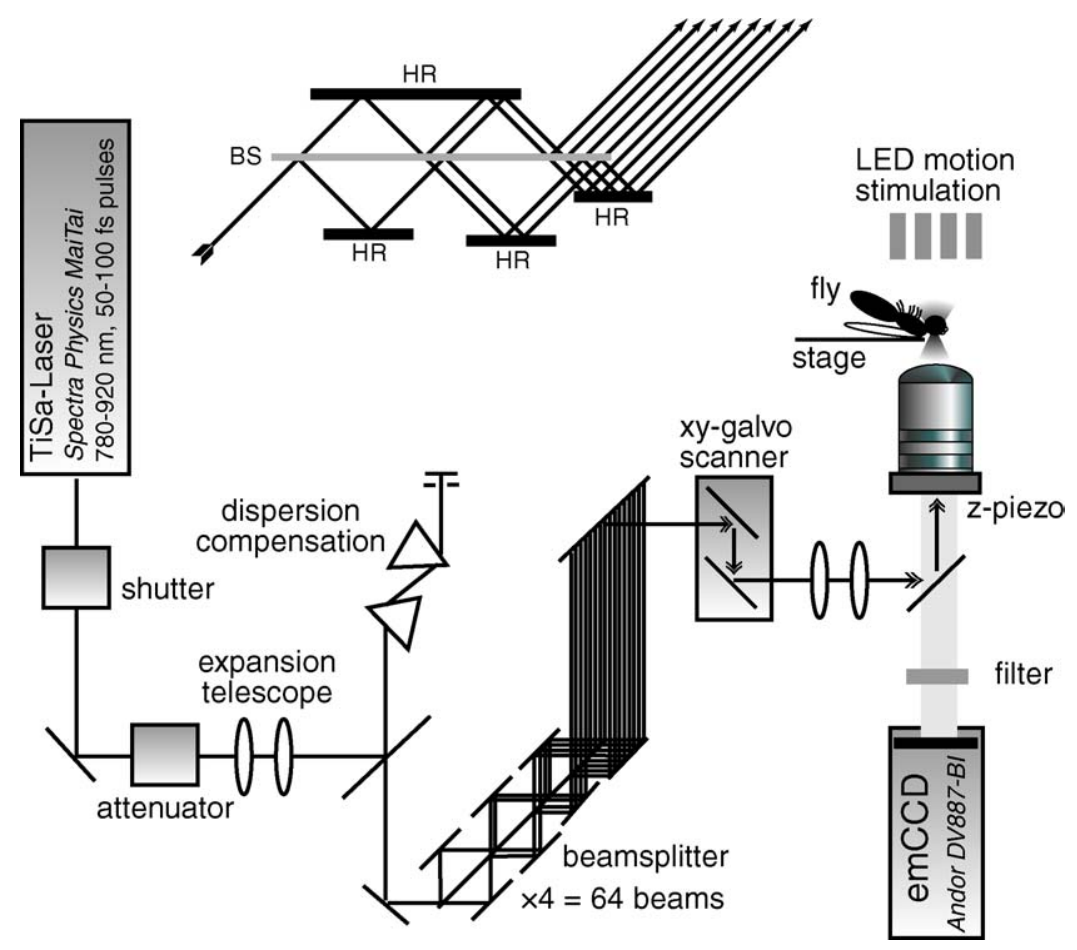

Fig. 2. Schematic of multiline TPLSM. Top: principle of the mirror-based beam multiplexer as first described by Nielsen et al. (2001). A linear array of in this case eight laser beams is generated by repeated separation at a 50\%-beamsplitter mirror (BS) and reflection at high-reflectivity mirrors (HR). Bottom: schematic of the components in the setup for multiline TPLSM. The laser beam of a tunable Ti:sapphire laser passes a shutter, an attenuator, a beam telescope to adjust the size of the beam, and a dispersion compensation consisting of a pair of prisms. The latter prevents elongation of laser pulses by compensating for the group velocity dispersion introduced by the glass in the optical path of the laser beam. The laser beam then enters the beam multiplexer which divides the laser beam into 64 beams. In the commercially available version of the beam multiplexer (LaVision BioTec, Bielefeld) used now in our setup the maximal number of beams can be reduced by software-controlled replacement of part of the beamsplitter mirror with a high-reflectivity mirror. However, in order to generate a beam array with widely separated laser foci (as used for the recording shown in Fig. 3B), manual blocking of some of the beams had to be used. Scan movements of the beam array are controlled by nonresonant $x y$-galvo-scan mirrors. Before entering the microscope (Olympus IX70), a second beam telescope is passed. The microscope long-distance water-immersion $40 \times /$ N.A. 0.8 objective was mounted on a z-piezo to control the focal plane for the acquisition of image stacks. The fly is fixed with bees wax at a small glass plate and mounted on the microscope stage to control its $x y$-position. Moving patterns are presented in the visual field of the fly at high contrast and fast update rate with a custom-built LED board. The microscope was not equipped with conventional epifluorescence illumination. However, delivery of excitation light (mercury lamp band-pass filtered at $420-480 \mathrm{~nm}$ ) to the sample via a lightguide (not shown in the diagram) turned out more practical than TPLSM to localize the dye-filled neuron at the beginning of an experiment. In the first case, a $465 \mathrm{~nm}$ beamsplitter and a $515 \mathrm{~nm}$ long-pass emission filter was used. In the case of TPLSM a $680 \mathrm{~nm}$ beamsplitter in combination with a TP-emission filter (short pass $700 \mathrm{~nm}$ ) was used. An EMCCD camera (Andor Ixon DV887BI) was installed for the detection of emission light.

Due to technical limitations, the use of imaging detection in TPLSM was up to now mainly restricted to applications which either provide high levels of emission light or do not require high temporal resolution (see e.g. Bewersdorf et al., 1998). In order to obtain highest possible sensitivity (and thus temporal resolution) even at the dim excitation light levels typical of in vivo imaging in intact animals, we used a back-illuminated CCD with quantum efficiency $>90 \%$ at $500-650 \mathrm{~nm}$ (i.e. in a range including the emission maxima of most calcium dyes) and variable electronmultiplying gain factor of up to 1000 (Andor iXon DV887BI) (Coates et al., 2004).

\subsection{Acquisition modes in multiline TPLSM}

We applied multiline TPLSM in two acquisition modes: in the first mode, called 'frame scan mode' throughout this study, the entire array of 64 laser beams was used to scan the sample in both $x$ - and $y$-directions. Thus, homogeneous illumination is elicited in the focal plane, given that lateral step size of the beam array is not too coarse (usually step sizes $\leq 400 \mathrm{~nm}$ were used). In Fig. 3A, an image sequence, acquired in 'frame scan mode' at a rate of $8.2 \mathrm{~Hz}$ from the presynaptic region of a VS neuron during presentation of pattern motion in the neuron's preferred direction is shown. In the colour-coded images, red and yellow indicate increases in the relative fluorescence $\left(\Delta F / F_{0}\right)$ of the calcium indicator dye, which are associated with a local rise in free intracellular calcium concentration.

In the 'frame scan mode' acquisition rates of up to approximately $20 \mathrm{~Hz}$ were feasible given sufficiently high dye staining intensity of the neurons. However, to speed up acquisition rates considerably, it is possible to record simultaneous line scans instead of entire images. This was done by restricting the scan movements of the laser beam array to the $x$-direction. Fig. 3B shows data from the same area as in A acquired in this 'multiple line scan' mode at a sampling rate of $89.3 \mathrm{~Hz}$. In order to prevent crosstalk between the laser beams, only eight widely separated beams (approximately $7 \mu \mathrm{m}$ distance) instead of the complete array of 64 beams were used in the example shown. It 
is also possible to use more beams with accordingly fewer distance between individual beams (data not shown). In the example shown in Fig. 3B four out of the eight lines hit neurites in the presynaptic region of the VS cell in the focal plane. For these multiple scan lines the fluorescence time courses can be evaluated by methods similar to those applied to single-line scan data.

\subsection{Spatio-temporal analysis of calcium dynamics by multiline TPLSM}

By multiline TPLSM simultaneous calcium signals at several neurites, which are too far apart from each other or too awkwardly arranged to be easily hit by a single scan line, could be compared in their fine-scale temporal dynamics. In the example shown in Fig. 3C (top), the calcium signal from one of the thin side branches was considerably faster in its rise and decline than signals from the main branch. This finding was corroborated by a comparison of calcium signals in different regions of interest in the image sequence recorded in the 'frame scan mode' at a slower temporal resolution (see Fig. 3C, bottom). Differences in surface area-to-volume ratio between neurites had been found to contribute to such differences in calcium dynamics (Kurtz, 2004). A potential functional significance might lie in the fact, that fast calcium dynamics in presynaptic endings helps to accelerate the control of transmitter release (see e.g. Callaway et al., 1993; Macleod et al., 2002; Regehr and Atluri, 1995).

By the use of confocal and TPLSM we were able to observe small neuritic protrusions in the axonal output region of VS neurons. These structures, which are reminiscent of dendritic spines in mammalian cortical neurons, have not been subject to functional imaging in TCs so far. Note, that it is currently unknown, whether these VS-cell 'spines' are purely post- or presynaptic, or both, as it is the case in olfactory bulb granule cell spines (Isaacson and Strowbridge, 1998). Since differential calcium regulation in spines and parent dendrites plays a major role in cortical spines (for review see Sabatini et al., 2001), we compared the calcium dynamics during visual stimulation between VS-cell 'spines' and the adjacent axonal trunk (Fig. 3D). In contrast to what might be expected from studies on mammalian pyramidal neurons, where spine calcium dynamics have been found to be faster in spines than in parent dendrites (Holthoff et al., 2002; Majewska et al., 2000) there were no obvious differences in calcium dynamics between the 'spines' and the parent axon or between individual 'spines' of the VS-neuron (see Fig. 3D).

\subsection{Sensitivity of the emission-light detection scheme}

In functional microscopy imaging speed depends essentially on how efficiently the light emitted by the sample is detected. This is in particular the case for imaging in living animals, where excitation intensity must be kept within strict limits to prevent photodamage. Although the long wavelengths used in TPLSM are in principle less harmful than the wavelengths used for onephoton excitation, photodamage is still an issue, in particular when trying to raise temporal resolution, because this makes high excitation power necessary (Hopt and Neher, 2001; Koester et al., 1999). For in vivo high-speed calcium imaging it is therefore crucial to use a highly-sensitive detection scheme.

The ability to detect the emission generated by single fluorophores can be used as a measure of detection efficiency. With our setup, single quantum dots (QDs) could easily be imaged. Even with exposure times in the range of a few $\mathrm{ms}$ the signals from single QDs rose above background noise (see Fig. 4A). In temporal image sequences, most of the fluorescent spots showed binary on-off responses ('blinking', see Fig. 4B). This clearly indicated, that the fluorescence emanated from single QDs and not from aggregates of several dots, because these would show intermediate brightness states.

In order to quantify the detection efficiency of our setup we estimated the photon emission rate of a single QD: first, our calculations clearly indicated that laser power was above saturating levels during the imaging of QDs. This is due to the extremely large two-photon absorbance cross-section of QDs (>30,000 GM for our type of QD, see Larson et al., 2003). The photon emission rate of a single QD can then be estimated based on the mean fluorescence lifetime of the QDs and the pulse repetition rate of the laser. The principle of this calculation is as follows: after a saturating pulse of laser power, only a certain fraction of QDs is back in the excitable state when the second laser pulse arrives, as is given by:

$F=\mathrm{e}^{-(t / \tau)}$,

with $t$ is the arrival time of second laser pulse $(12.5 \mathrm{~ns}$ for a $80 \mathrm{MHz}$ laser) and $\tau$ is the mean fluorescence life time of QDs ( $\tau$ is $14.5 \mathrm{~ns}$ for our type of QD; A. Biebricher, C. Müller, P. Tinnefeld, M. Sauer, personal communication).

Next, the fraction of QDs which is ready to be excited by the third laser pulse is calculated by the same exponential law, but starting with 1-F QDs. When repeating this procedure and summing up the results over sufficiently many steps, we obtained $34.3 \mathrm{~ns}$ as the mean excitation interval, i.e. a mean excitation rate of $29.2 \mathrm{MHz}$. Normalized by the specific quantum yield of the QDs, which is about $40 \%$ (A. Biebricher, C. Müller, P. Tinnefeld, M. Sauer, personal communication), the effective excitation rate is $11.7 \mathrm{MHz}$. This value has to be multiplied by the fraction of time the laser sweeps over the QD, i.e. when the QD lies within the laser focus. With a full-width at half-maximum (FWHM) of the point-spread function (PSF) of $560 \mathrm{~nm}$ (see below) and a scan path of $40 \mu \mathrm{m}$, this is the case in about $1.4 \%$ of the scan time. This estimation yields a mean photon emission rate of $164 \mathrm{kHz}$ for a single $\mathrm{QD}$, which is equivalent to about 820 photons per $5 \mathrm{~ms}$ interval, the shortest CCD exposure time we used for QD imaging (see Fig. 4A).

In a second calculation, we estimated how many photons contributed to the image of a single QD on our CCD sensor: the intensity above background integrated over single QDs was $15250 \pm 2919$ (mean \pm S.D., $n=6$ ) counts per second on average. By taking into account the CCD's gain applied in these measurements (about 75 -fold as determined in calibration measurements), a CCD sensitivity of 23 electrons per analog-digital count and a quantum detection efficiency of $85 \%$ (both values given by manufacturer), a value of about $5500 \pm 1053$ photons/s 
(A)

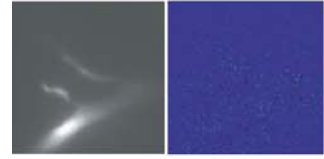

$10 \overline{\mu m}$

(B)

4

3

2

1

$$
1
$$
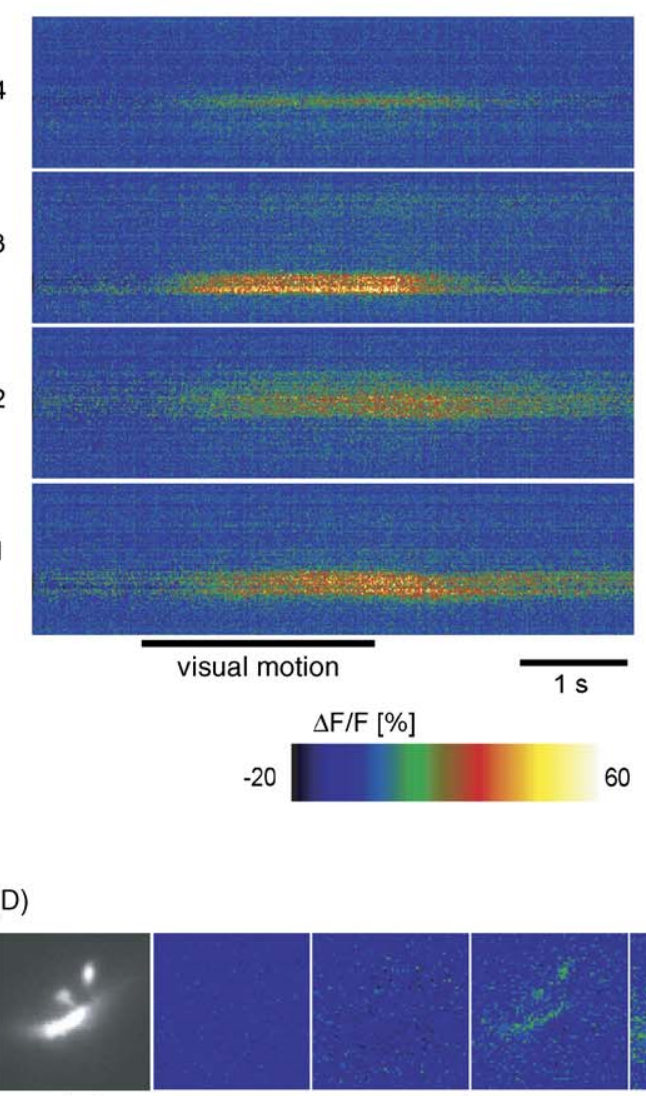

\section{0}

(D)
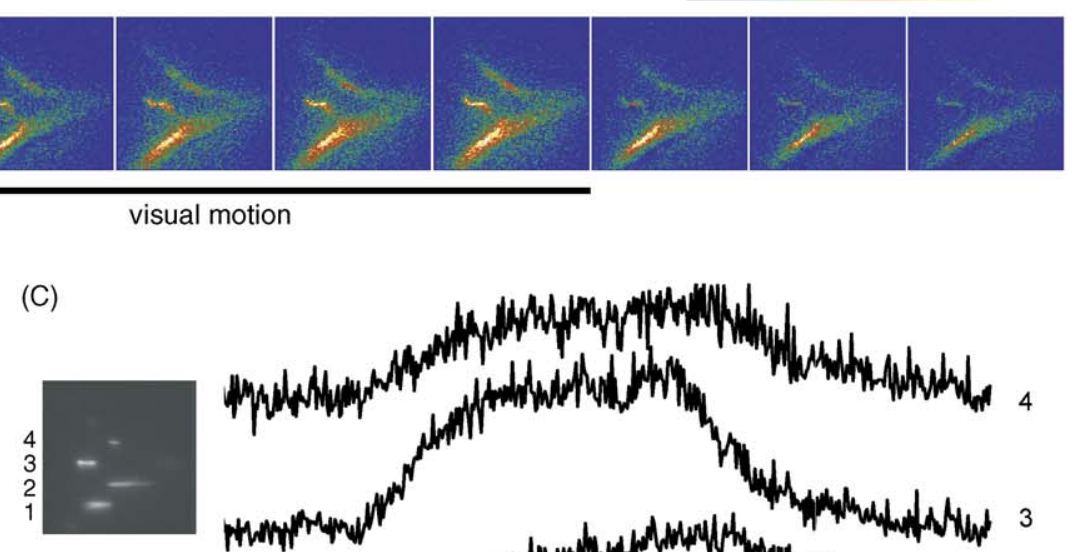

visual motion

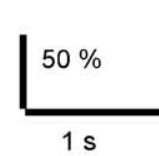

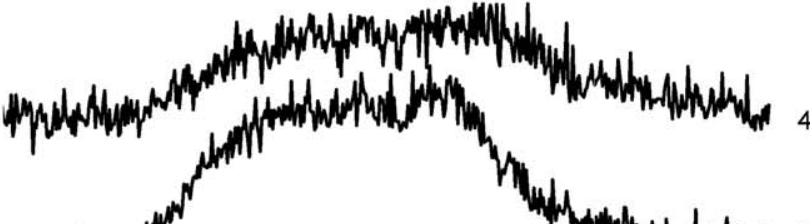

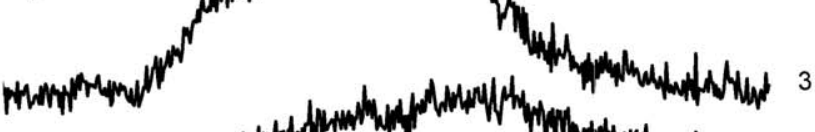

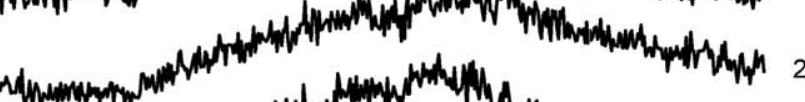
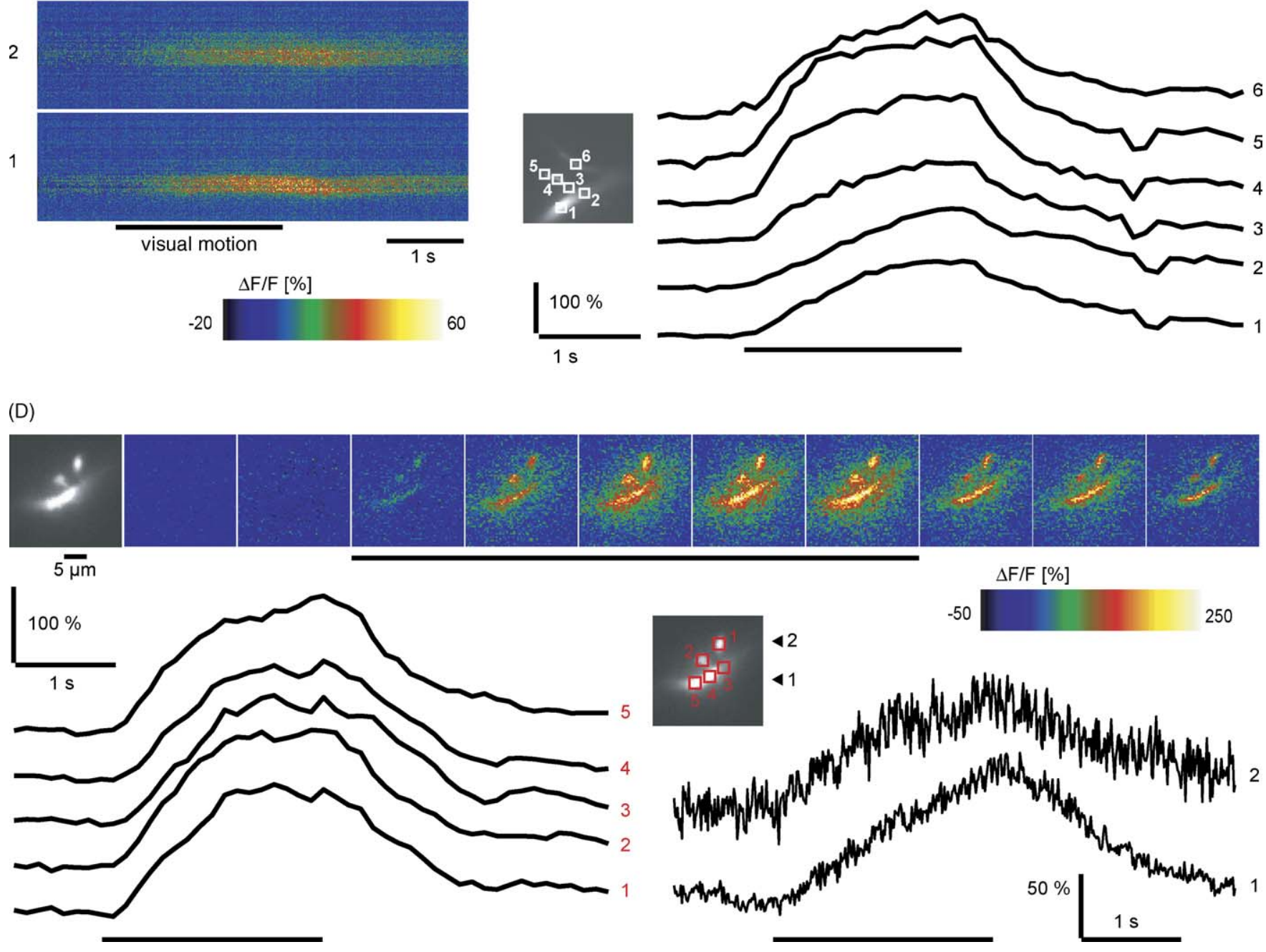
(A)

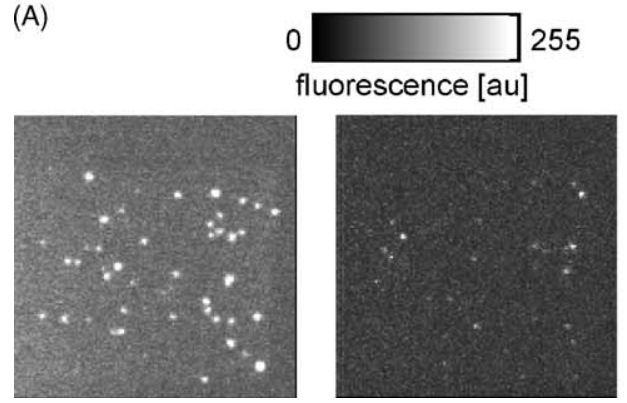

(B)

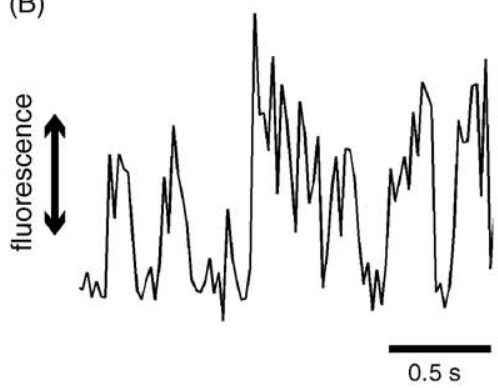

(C)

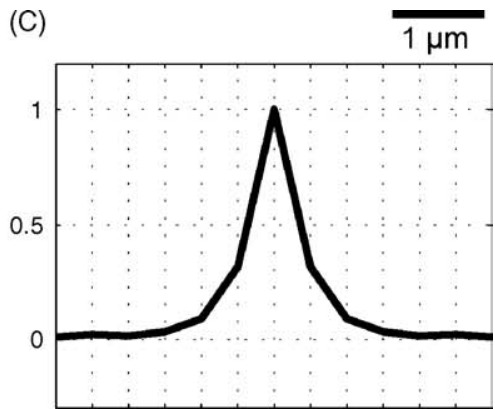

Fig. 4. Visualization of single quantum dots (QDs). (A) Images of QDs (Qdot 605 Streptavidin Conjugate adsorbed on dry cover slides) acquired with multiline TPLSM in 'frame scan mode' at $800 \mathrm{~nm}$ with exposure times of $100 \mathrm{~ms}$ (left) and $5 \mathrm{~ms}$ (right). (B) Fluorescence collected from a single QD. On-off responses ('blinking') are visible in the fluorescence time course. (C) Mean point-spread function of single QDs. The full-width at half-maximum (FWHM) was 560 nm with a long-distance water-immersion $40 \times /$ NA 0.8 objective. Vertical grid lines indicate the effective pixel size of the CCD-camera $(400 \mathrm{~nm})$.

is obtained for single QDs, equivalent $27.5 \pm 5.3$ photons in $5 \mathrm{~ms}$ exposures. A comparison of this value with the number of photons emitted by single QDs as estimated above, yields an overall detection efficiency of $3-4 \%$, which appears to be in a plausible range. Major factors influencing detection efficiency are the angular acceptance of the microscope objective and light loss inside the objective (LUMPlanFl/IR $40 \times / 0.80$, transmission $85 \%$ at $500 \mathrm{~nm}$, value given by manufacturer), at the beamsplitter and the emission filter (680DCSPXR and E700SP-2P, AHF, transmission of both $>90 \%$ at $500 \mathrm{~nm}$ ) and at several additional air-glass interfaces inside the microscope and at the CCD housing.

\subsection{Spatial resolution of the setup for multiline TPLSM}

Detection of tiny structures in the nm-range like QDs does, of course, not imply much about spatial resolution. Rather, it may provide an indicator of sensitivity (see above). However, since structures of such small size can be regarded pointlike light sources, mapping their PSF directly yields a measure of the setup's upper limit of spatial resolution. We determined the FWHM of the mean PSF. It was $560 \mathrm{~nm}$ with the long-distance water-immersion objective lens (Olympus LUMPlanFI/IR $40 \times / 0.80$ ) used in our in vivo preparation for calcium imaging (see Fig. 4C). Of course, objectives with higher magnification and numerical aperture provided considerably better spatial resolution. For example, a FWHM of $350 \mathrm{~nm}$ was obtained using a Olympus UPlanAPO $60 \times$, NA $1.2 \mathrm{~W}$ together with the internal $1.5 \times$ magnification lens at the Olympus IX70 microscope.

The absolute pixel size of the CCD in our two-photon setup is $16 \mu \mathrm{m} \times 16 \mu \mathrm{m}$, which yields $400 \mathrm{~nm} \times 400 \mathrm{~nm}$ effective size with 40 -fold magnification. Therefore, we evaluated whether the spatial resolution of our setup may be limited by the pixel size of the CCD camera rather than by the microscope optics itself. For two reasons we conclude that spatial resolution would be increased to some extend by the use of a CCD with smaller pixels (note however, that smaller pixel size might degrade CCD sensitivity).

First, the theoretical resolution limit for two-photon excitation can be approximated by (Zipfel et al., 2003):

$\mathrm{FWHM}=\frac{0.38 \lambda}{\mathrm{NA}^{0.91}}$

with $\lambda$ is the wavelength and NA is the numerical aperture; for $\mathrm{NA}>0.7$.

With NA $=0.8$ and $\lambda=820 \mathrm{~nm}$ application of this formula yields a FWHM of $382 \mathrm{~nm}$, which is slightly below the CCD pixel size.

Second, we performed an equivalent analysis with a confocal microscope (Leica TCS SP 2, objective: HCX APO L U-V-I $40 \times / 0.80$ ). In this case, fluorescent microspheres (size $40 \mathrm{~nm}$, excitation/emission 488/685) were used instead of QDs. The FWHM of the PSF was $420 \mathrm{~nm}$ (data not shown), which is slightly above pixel size of the CCD in the two-photon setup.

Fig. 3. Calcium signals at the presynaptic region of a VS2-neuron (see Fig. 1) monitored with multiline TPLSM during visual stimulation. (A) A sequence of $128 \times 128$ pixel images was acquired at a rate of 8.2 frames/s during presentation of motion in the neuron's preferred direction (only every fourth frame is shown). The array of 64 laser beams was scanned in $x$ - and $y$-direction, thus generating homogeneous illumination of the focal plane ('frame scan mode'). The neuron was filled with a mixture of two calcium dyes, Oregon-Green 1 and Fluo 4 (see Table 1). This procedure allowed us to overcome the low baseline fluorescence of Fluo 4 , while still being able to profit to some extent from its high dynamic range. Relative increases in fluorescence of the calcium dyes $\left(\Delta F / F_{0}\right)$ excited at $820 \mathrm{~nm}$ indicate a rise in the concentration of intracellular free calcium. Such increases appear red and yellow in the colour-coded images. (B) Multiple line scans were recorded simultaneously by scanning eight laser beams along the $x$-dimension. Arrowheads besides the 2D-image indicate the position where four of these beams hit neuronal structures in the focal plane (top left). Top right: corresponding linescan image. Bottom: colour-coded linescan data over time acquired at a temporal resolution of $89.3 \mathrm{~Hz}$ along the four lines during visual stimulation (as in A). Note, that the spatial axis has been tilted relative to the images shown above: top in the linescans corresponds to right in the images. (C) Top: time courses of the calcium signals $\left(\Delta F / F_{0}\right)$ calculated from the 'multiple line scan' data (see B). Bottom: time courses calculated from the 'frame scan' data (see A) for several regions of interest. (D) Calcium imaging at 'spine'-like structures in the axonal output region of the same VS2-neuron. Top: colour-coded image sequence of the calcium signal (as in A). Bottom left: $\Delta F / F_{0}$ time courses calculated from the 'frame scan' image series in different regions, as indicated by the boxes in the inset. Bottom right: $\Delta F / F_{0}$ time courses calculated from two line scans acquired in the 'multiple line scan' mode at positions as indicated by the arrowheads in the inset. Note, that a background subtraction was applied to all fluorescence data shown. For illustrative reasons, background subtraction in the colour-coded images was performed with values slightly below actual background intensity to reduce noise in areas with low intensity. 


\subsection{Spatial resolution with imaging versus point-scanning detection mode}

Spatial resolution as determined by imaging of QDs can, if at all, only be reached at superficial tissue layers, but not deep inside scattering tissue. This is due to the fact that for all the measurements shown up to here we used imaging detection instead of a point-scanning detection scheme, i.e. we took one image for one or multiple sweeps of the laser rather than for every laser position. With a point-scanning detection scheme, scattered emission photons can be collected and reassigned to the laser focus during image reconstruction. This is not possible when imaging detection with a CCD is applied as in our setup: scattering of photons on their way from the laser focus to the detector will deteriorate spatial resolution to some extend. Important to note, one major advantage of TPLSM, namely confinement of excitation to the laser focus, remains unaffected by the detection scheme used. Moreover, in the 'multiple line scan' mode only resolution along lines, but not lateral resolution is affected, if the spacing between individual lines is large enough to avoid an overlap of the signals.

In order to determine directly in how far spatial resolution is degraded by scattering of emission photons in compact brain tissue, we applied both a point-scanning and an imaging detection mode at our setup. This was done by scanning short lines at a low scan frequency during fast image acquisition. With $15 \mu \mathrm{m}$ lines scanned at $2 \mathrm{~Hz}$ and a CCD acquisition rate of $97 \mathrm{~Hz}$, every image contains the signal gathered along a $313 \mathrm{~nm}$ segment of the scan path. Thus, the fluorescence time course calculated from the image sequence can be used to draw the spatial intensity profile of structures crossed by the scan line. In contrast to this pointscanning detection scheme, an image averaged over the entire sequence directly yields the spatial profile corresponding to the imaging detection mode. Thus, one and the same data set could be used to obtain linescan profiles which are in the first case unaffected by scattering and in the second case blurred by scattering of photons in the tissue after their emission from the laser focus.
Spatial intensity profiles of small-diameter neurites of fly TCs appeared slightly narrower and had steeper flanks, when extracted from the image sequence according to the pointscanning detection scheme than when taken from the average image. In the example shown in Fig. 5A, the FWHM of the normalized intensity profile was $2.80 \mu \mathrm{m}$ for point-scanning detection (broken line). For imaging detection the FWHM of the corresponding profile was $3.90 \mu \mathrm{m}$ when the CCD row containing the brightest signal was taken from the average image (Fig. 5A, thin line, see arrowhead in inset). A slightly broader FWHM, $3.95 \mu \mathrm{m}$, was obtained when the signal was vertically averaged over the 10 brightest CCD rows, which may be used to increase signal-to-noise ratio (thick line). Unlike PSFs, line scans of neurites do not yield direct measures of spatial resolution. However, the absolute difference between the FWHM of spatial intensity profiles determined with the two detection modes $(\triangle \mathrm{FWHM})$ provides a relative measure of the difference in spatial resolution. For several line scans at the region shown in Fig. 5A, $\triangle$ FWHM amounted to $0.88 \pm 0.47$ and $1.02 \pm 0.32 \mu \mathrm{m}$ (mean \pm S.D., $n=7$ ), when taking single $\mathrm{CCD}$ rows and averages over the 10 brightest CCD rows of the average image, respectively. This indicates that, first, point-scanning detection consistently provides superior spatial resolution than imaging detection. Second, spatial resolution with imaging detection may be further reduced slightly by signal averaging perpendicular to the scan line. $\triangle F W H M$ values determined for two further neurites were $0.44 \pm 0.34(n=7)$ and $0.92 \pm 0.31(n=9)$ when single CCD rows were evaluated during imaging detection and $0.92 \pm 0.47(n=7)$ and $1.22 \pm 0.30(n=9)$ when averages over $10 \mathrm{CCD}$ rows were taken.

With point-scanning detection, spatial resolution may be further improved by decreasing spatial bin size. We realized this by decreasing the length of the scan line while maintaining scan frequency and image acquisition rate. The FWHMs of spatial profiles of the region shown in Fig. 5A acquired at bin sizes of 313 and $157 \mathrm{~nm}$ were $2.80 \pm 0.26 \mu \mathrm{m}(n=7)$ and $2.71 \pm 0.57 \mu \mathrm{m}$ $(n=4)$, respectively. These values indicate that, in the range of
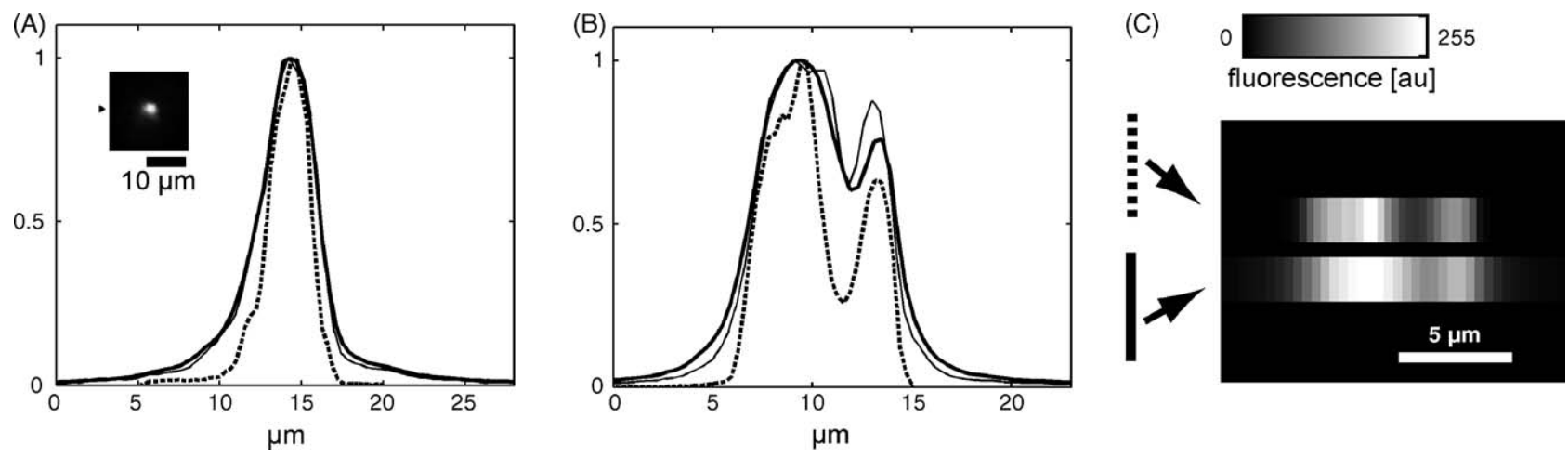

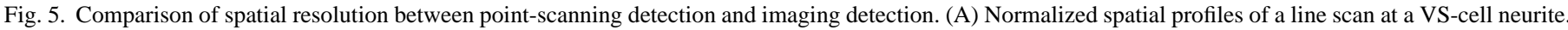

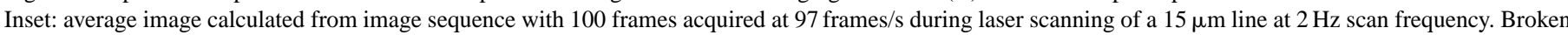

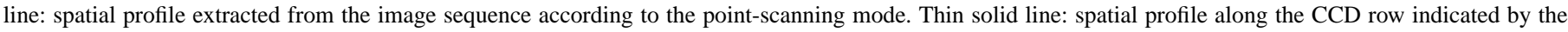

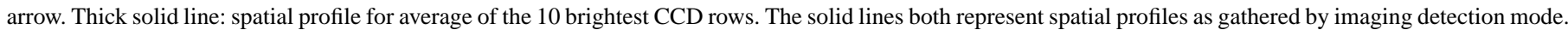

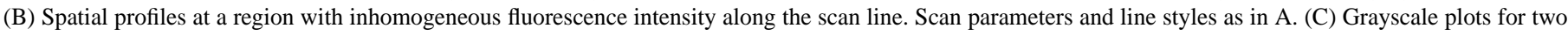

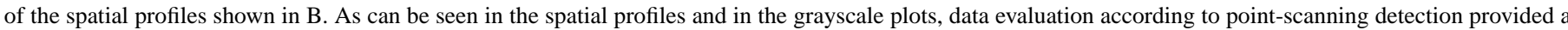
clearer resolution of the gap in fluorescence intensity along the scan line than data evaluation according to imaging detection. 
bin sizes used, spatial resolution during point-scanning detection was not limited by bin size. Therefore, it seems fairly unlikely, that much of the difference in spatial resolution between imaging mode and point-scanning mode was due to the larger bin size during imaging mode (which is in the latter equivalent to CCD pixel size, $400 \mathrm{~nm}$ ) instead of scattering.

The difference in spatial resolution between imaging and point-scanning becomes clearly evident when neurites have a spatially inhomogeneous intensity along the scan line. One such example is shown in Fig. 5B and C: a deep gap in the intensity profile could be resolved when line scans were performed in the point-scanning mode (Fig. 5B, broken line), but was less pronounced with imaging mode (thin line: single row of the CCD image, thick line: average of 10 rows of the CCD image).

\section{Discussion}

In this study, we further developed multiline TPLSM (Nielsen et al., 2001) by using a fast and sensitive CCD detector and tested its applicability to functional in vivo imaging. The use of a CCD detector gives us the opportunity to use multiline TPLSM in two acquisition modes, which we characterized in the present study with respect to spatial and temporal resolution: (1) imaging detection is fast with up to about $100 \mathrm{~Hz}$ temporal resolution but suffers from some loss in spatial resolution under realistic conditions in scattering tissue, (2) point-scanning detection exploits the optimal spatial resolution that can be reached in TPLSM but is considerably slower, with acquisition rates of several Hz. Thus, when performing functional imaging with multiline TPLSM imaging detection can be used to reach a high temporal resolution and can be switched immediately without modifications to the setup to point-scanning detection whenever acquisition speed is less critical than spatial resolution.

In non-scattering samples, even with the use of imaging detection spatial resolution of our multiline TPLSM setup comes close to state-of-the-art confocal microscopes. Thus, as is suggested by imaging single QDs, submicrometer resolution can be reached with multiline TPLSM and imaging detection in superficial tissue layers. In contrast, spatial resolution in compact tissue is degraded by scattering of emitted photons if imaging detection instead of a point-scanning detection is used. Since both detection modes are feasible with our setup, we could directly compare their spatial resolution, not only in one and the same sample, but even with identical data sets. The FWHM of spatial profiles of neurites in our preparation differed by about $1 \mu \mathrm{m}$ between imaging and point-scanning detection. This value indicates a considerable advantage of point-scanning detection over imaging detection in biological tissue. Apart from sample structure, an important factor influencing scattering is imaging depth. The neurites at which spatial resolution was tested in the present study were all in a similar depth of 30-60 $\mu \mathrm{m}$ below tissue surface. The effect of scattering, and thus the difference in spatial resolution between point-scanning and imaging detection are expected to be less relevant in more superficial imaging regions and more pronounced deeper inside the tissue. The TCs of the fly arborize in an almost two-dimensional layer, relatively shallow below the brain surface. To clarify in how far scattering is influ- enced by imaging depth, other preparations with neurons that descent deeper into the neural tissue, like the pyramidal neurons of mammalian cortex, are much more suitable.

We optimized our multiline TPLSM setup for detection sensitivity, in particular by the use of a new type of CCD with the highest possible quantum efficiency combined with electronmultiplying capacity (Coates et al., 2004). Although sensitivity is difficult to quantify, here it is exemplified by the detection of single QDs even at very short exposure times (down to $5 \mathrm{~ms}$ ). Detection sensitivity is a critical parameter in particular for functional in vivo imaging: high excitation power cannot compensate for poor sensitivity, because it would lead to photobleaching or, even worse, photodamage. For single-beam TPLSM photodamage was found to occur already at levels as low as $10 \mathrm{~mW}$ incident laser power at the specimen (Hopt and Neher, 2001; Kalb et al., 2004; Koester et al., 1999). With excitation power distributed on multiple laser foci, total power can be expected to be much higher without reaching harmful levels. Although we did not systematically determine thresholds to harmful power levels, we neither observed photodamage nor considerable photobleaching when incident laser power at the specimen was estimated to be as high as $308 \mathrm{~mW}$. Our estimation is based on a measured value of $410 \mathrm{~mW}$ at the back focal plane of the objective lens, a $75 \%$ transmission of the objective lens at $820 \mathrm{~nm}$ (value given by manufacturer) and neglecting power loss resulting from slight overillumination of the objective's back focal aperture. Note, that this corresponded to only about half of the available laser power, leaving a margin for imaging in deeper layers of tissue or in even more opaque preparations.

The major general advantage of our setup compared to conventional laser-scanning confocal or two-photon microscopes is that it becomes feasible to get multiple simultaneous linescans from cellular structures which are separated too far to be covered by a single line. In the present study, this was demonstrated for calcium imaging in motion-sensitive neurons of the fly. In numerous other preparations, particularly in neuroscience, multiline TPLSM can also be expected to offer considerable advantages. For example, when studying plasticity-related processes in spines on mammalian cortical neurons, it is often necessary to compare calcium signals at spine head, spine neck and parent dendrite during synaptic activation or during actionpotential backpropagation (see e.g. Muller and Connor, 1991; Sabatini et al., 2002). Such comparisons, and even comparisons between neighbouring spines, would become more direct by the use of multiline TPLSM instead of single-line scans.

\section{Acknowledgements}

The authors thank Christian Müller for his contribution to the calculation of mean point-spread functions and Martin Egelhaaf for many fruitful discussions and for comments on the manuscript.

\section{References}

Bewersdorf J, Pick R, Hell SW. Multifocal multiphoton microscopy. Opt Lett 1998;23:655-7. 
Borst A, Egelhaaf M. In vivo imaging of calcium accumulation in fly interneurons as elicited by visual motion stimulation. Proc Natl Acad Sci USA 1992;89:4139-43.

Borst A, Haag J. Neural networks in the cockpit of the fly. J Comp Physiol [A] 2002;188:419-37.

Callaway JC, Lasser-Ross N, Stuart AE, Ross WN. Dynamics of intracellular free calcium concentration in the presynaptic arbors of individual barnacle photoreceptors. J Neurosci 1993;13:1157-66.

Coates CG, Denvir DJ, McHale NG, Thornbury KD, Hollywood MA. Optimizing low-light microscopy with back-illuminated electron-multiplying charge-coupled device: enhanced sensitivity, speed, and resolution. J Biomed Opt 2004;9:1244-52.

Denk W, Detwiler PB. Optical recording of light-evoked calcium signals in the functionally intact retina. Proc Natl Acad Sci USA 1999;96:7035-40.

Dürr V, Egelhaaf M. In vivo calcium accumulation in presynaptic and postsynaptic dendrites of visual interneurons. J Neurophysiol 1999;82:3327-38.

Dürr V, Kurtz R, Egelhaaf M. Two classes of visual motion-sensitive interneurons differ in direction and velocity dependency of in vivo calcium dynamics. J Neurobiol 2001;46:289-300.

Egelhaaf M, Borst A. A look into the cockpit of the fly: visual orientation, algorithms, and identified neurons. J Neurosci 1993;13:4563-74.

Egelhaaf M, Borst A, Warzecha AK, Flecks S, Wildemann A. Neural circuit tuning fly visual neurons to motion of small objects. II. Input organization of inhibitory circuit elements revealed by electrophysiological and optical recording techniques. J Neurophysiol 1993;69:340-51.

Egelhaaf M, Grewe J, Karmeier K, Kern R, Kurtz R, Warzecha AK. Novel approaches to visual information processing in insects: case studies on neuronal computations in the blowfly. In: Christensen TA, editor. Methods in insect sensory neuroscience. Boca Raton: CRC Press; 2005. p. $185-212$.

Egelhaaf M, Hausen K, Reichardt W, Wehrhahn C. Visual course control in flies relies on neuronal computation of object and background motion. Trends Neurosci 1988;11:351-8.

Egelhaaf M, Kern R, Krapp HG, Kretzberg J, Kurtz R, Warzecha A-K. Neural encoding of behaviourally relevant visual-motion information in the fly. Trends Neurosci 2002;25:96-102.

Euler T, Detwiler PB, Denk W. Directionally selective calcium signals in dendrites of starburst amacrine cells. Nature 2002;418:845-52.

Haag J, Borst A. Recurrent network interactions underlying flow-field selectivity of visual interneurons. J Neurosci 2001;21:5685-92.

Haag J, Denk W, Borst A. Fly motion vision is based on Reichardt detectors regardless of the signal-to-noise ratio. Proc Natl Acad Sci USA 2004;101:16333-8.

Halbhuber KJ, König K. Modern laser-scanning microscopy in biology, biotechnology and medicine. Ann Anat 2003;185:1-20.

Helmchen F, Denk W. New developments in multiphoton microscopy. Curr Opin Neurobiol 2002;12:593-601.

Helmchen F, Waters J. Ca2+ imaging in the mammalian brain in vivo. Eur J Pharmacol 2002;447:119-29.

Holthoff K, Tsay D, Yuste R. Calcium dynamics of spines depend on their dendritic location. Neuron 2002;33:425-37.

Hopt A, Neher E. Highly nonlinear photodamage in two-photon fluorescence microscopy. Biophys J 2001;80:2029-36.

Horstmann W, Egelhaaf M, Warzecha AK. Synaptic interactions increase optic flow specificity. Eur J Neurosci 2000;12:2157-65.
Isaacson JS, Strowbridge BW. Olfactory reciprocal synapses: dendritic signaling in the CNS. Neuron 1998;20:749-61.

Kalb J, Nielsen T, Fricke M, Egelhaaf M, Kurtz R. In vivo two-photon laser-scanning microscopy of $\mathrm{Ca}(2+)$ dynamics in visual motion-sensitive neurons. Biochem Biophys Res Commun 2004;316:341-7.

Koester HJ, Baur D, Uhl R, Hell SW. Ca2+ fluorescence imaging with picoand femtosecond two-photon excitation: signal and photodamage. Biophys J 1999;77:2226-36.

Krapp HG, Hengstenberg R. Estimation of self-motion by optic flow processing in single visual interneurons. Nature 1996;384:463-6.

Kurtz R. Ca2+ clearance in visual motion-sensitive neurons of the fly studied in vivo by sensory stimulation and $\mathrm{UV}$ photolysis of caged $\mathrm{Ca} 2+\mathrm{J}$ Neurophysiol 2004;92:458-67.

Kurtz R, Dürr V, Egelhaaf M. Dendritic calcium accumulation associated with direction-selective adaptation in visual motion-sensitive neurons in vivo. J Neurophysiol 2000;84:1914-23.

Kurtz R, Warzecha AK, Egelhaaf M. Transfer of visual motion information via graded synapses operates linearly in the natural activity range. J Neurosci 2001;21:6957-66.

Larson DR, Zipfel WR, Williams RM, Clark SW, Bruchez MP, Wise FW, et al. Water-soluble quantum dots for multiphoton fluorescence imaging in vivo. Science 2003;300:1434-6.

Leveque-Fort S, Fontaine-Aupart MP, Roger G, Georges P. Fluorescencelifetime imaging with a multifocal two-photon microscope. Opt Lett 2004;29:2884-6.

Macleod GT, Hegstrom-Wojtowicz M, Charlton MP, Atwood HL. Fast calcium signals in Drosophila motor neuron terminals. J Neurophysiol 2002;88:2659-63.

Majewska A, Brown E, Ross J, Yuste R. Mechanisms of calcium decay kinetics in hippocampal spines: role of spine calcium pumps and calcium diffusion through the spine neck in biochemical compartmentalization. J Neurosci 2000;20:1722-34

Muller W, Connor JA. Dendritic spines as individual neuronal compartments for synaptic $\mathrm{Ca} 2+$ responses. Nature 1991;354:73-6.

Nakano A. Spinning-disk confocal microscopy - a cutting-edge tool for imaging of membrane traffic. Cell Struct Funct 2002;27:349-55.

Nielsen T, Fricke M, Hellweg D, Andresen P. High efficiency beam splitter for multifocal multiphoton microscopy. J Microsc 2001;201:36876.

Passaglia CL, Troy JB. Impact of noise on retinal coding of visual signals J Neurophysiol 2004;92:1023-33.

Regehr WG, Atluri PP. Calcium transients in cerebellar granule cell presynaptic terminals. Biophys J 1995;68:2156-70.

Sabatini BL, Maravall M, Svoboda K. $\mathrm{Ca}(2+)$ signaling in dendritic spines. Curr Opin Neurobiol 2001;11:349-56.

Sabatini BL, Oertner TG, Svoboda K. The life cycle of $\mathrm{Ca}(2+)$ ions in dendritic spines. Neuron 2002;33:439-52.

Single S, Borst A. Dendritic integration and its role in computing image velocity. Science 1998;281:1848-50.

Warzecha AK, Egelhaaf M. Variability in spike trains during constant and dynamic stimulation. Science 1999;283:1927-30.

Wright SJ, Wright DJ. Introduction to confocal microscopy. Meth Cell Biol 2002;70:1-85

Zipfel WR, Williams RM, Webb WW. Nonlinear magic: multiphoton microscopy in the biosciences. Nat Biotechnol 2003;21:1369-77. 